\title{
Cardiac Valve Annulus Manual Segmentation Using Computer Assisted Visual Feedback in Three-Dimensional Image Data
}

\author{
Ramón Casero, Rebecca A.B. Burton, T. Alexander Quinn, Christian Bollensdorff, Patrick Hales, \\ Jürgen E. Schneider, Peter Kohl and Vicente Grau
}

\begin{abstract}
Annulus manual segmentation is an important tool for the study of valve anatomy and physiology, for the four main valves of the heart (mitral, tricuspid, aortic and pulmonary). In this paper we review two traditional manual segmentation approaches: slice-by-slice and interpolating a sparse set of landmarks with a spline curve. We propose a new Spline Tool for the open source software platform Seg3D, that is fast and improves spatial coherence by providing visual feedback of the segmentation in real time. The Spline Tool was tested successfully on 14 rat hearts, on all four valves.
\end{abstract}

\section{INTRODUCTION}

There are four main cardiac valves in the heart, two located between the atria and ventricles (mitral valve on the left side of the heart, tricuspid valve on the right), and two between the ventricles and the arteries leaving the heart (aortic valve on the left, pulmonary valve on the right).

Each valve consists of 2 or 3 cusps, or leaflets, that cover its opening, surrounded by a ring of connective tissue, or annulus. Shape and motion analysis of the annulae is important to obtain an insight into valve function, pathology and optimal surgery planning and treatment. All four annulae form part of the atrioventricular surface, that electrically insulates the atria from the ventricles. Thus, annulae segmentation can provide an initial estimate of the atrioventricular surface. Finally, the annulae provide natural boundaries for cavity segmentation.

Most studies of annular shape rely on expert manual segmentation [1], [3], [4], [6], [8], [10], [16] (mitral), [2], [7], [15] (tricuspid). A few methods for semi-automatic segmentation of valve annulae have been proposed in the last decade, e.g. [11], [13], [18], but manual segmentations have still been used for validation as a pseudo criterion (gold) standard. Therefore, annulus manual segmentation remains the dominating tool for the study of valve anatomy and physiology.

Annulae have usually been approximated in the literature by a closed curve, e.g. [18], or a toroid-like solid obtained

R. Casero is at the Computational Biology Group, Computing Laboratory, University of Oxford, Wolfson Building, Parks Rd, Oxford OX1 3QD, UK ramon.casero@comlab.ox.ac.uk.

R.A.B. Burton, T.A. Quinn, C. Bollensdorff and P. Kohl are at the Cardiac Mechano-Electric Feedback lab, Department of Physiology, Anatomy and Genetics, University of Oxford, Sherrington Building, Parks Rd, Oxford OX1 3PT, UK.

P. Hales and J.E. Schneider are at the Department of Cardiovascular Medicine, Wellcome Trust Centre for Human Genetics, University of Oxford, Roosevelt Dr, Oxford OX3 7BN, UK.

V. Grau is at the Institute of Biomedical Engineering, Department of Engineering Science, University of Oxford, and the Oxford e-Research Centre, University of Oxford, Parks Rd, Oxford OX1 3QD, UK. from the concatenation of disks or spheres in each slice [8]. The curve must be allowed to be nonplanar, as valves such as the mitral have an annulus with a saddle shape [3], [4], the tricuspid annulus has a more planar saddle shape and is rounder in the projected plane [7], [15], and the aortic annulus has a three pronged crown-like circlet shape [9].

Regarding imaging modalities, echocardiography has been historically preferred in the literature for annulus segmentation, even in recent publications [16], [18], as it has good temporal resolution, is safe, cheap and non-invasive. However, if motion analysis is sacrificed, three-dimensional (3D) Magnetic Resonance Imaging (MRI) provides much better image quality than echocardiography, principally because of higher spatial resolution and lack of speckle texture.

In this paper we review two traditional manual segmentation methods, slice-by-slice and interpolating a sparse set of landmarks with a spline curve. We find both approaches of limited utility and propose a new tool for the open source software platform Seg3D [19]. The new Spline Tool combines the speed of the sparse set of landmarks approach with improved spatial coherence provided by visual feedback of the segmentation in real time. We use very high resolution 3D MRI data obtained by state of the art wet lab and imaging techniques [14], [12], [17].

\section{DATA}

\section{A. Wet-Lab Methods}

All investigations reported in this study conformed to the UK Home Office guidance on the Operation of Animals (Scientific Procedures) Act of 1986. Sprague Dawley rat $(\approx 300 \mathrm{~g}, \mathrm{n}=14)$ hearts were excised and swiftly mounted to a Langendorff system for coronary perfusion with normal tyrode [14]. The hearts were cardioplegically arrested with high $\mathrm{K}^{+}(20 \mathrm{mM})$ solution in their slack (resting) state and fixed via coronary perfusion. Fixation was achieved by slowly perfusing the heart with Karnovsky fixative [14], being careful to avoid excessive pressure gradients, which have been seen to cause volume overload in the ventricles. Hearts were left overnight in fixative containing $4 \mathrm{mM}$ gadodiamide contrast agent, then washed for $30 \mathrm{~min}$ in sodium cacodylate buffer, and embedded in bubble-free $2 \%$ low melting agar (containing $4 \mathrm{mM}$ gadodiamide).

\section{B. Anatomical Imaging}

Anatomical MRI scans were performed using a Varian 9.4 T (400 MHz) MR system (Varian Inc, Palo Alto, CA), and a birdcage coil with an inner diameter of $28 \mathrm{~mm}$ (Rapid 
Biomedical, Wurzburg, Germany) to transmit / receive the NMR signals. A 3D gradient echo pulse sequence was used for data acquisition [12], [17], with a total scan time of 12 hours. Data were acquired at a typical experimental resolution of $43 \times 43 \times 19 \mu \mathrm{m}$, which was zero filled and written to a stack of TIFF images with a final resolution of $25.4 \mu \mathrm{m}$ in-plane, $12.7 \mu \mathrm{m}$ inter-plane. Fig. 1 shows three orthogonal sample slices.

\section{METHODS}

\section{A. Software platform}

All the data were visualized and segmented using Seg3D [19], an open source (MIT licence) software application developed in C++. Seg3D allows to load a 3D image volume in a popular format, e.g. MetaImage or NRRD, and scroll through the volume using 2 long-axis planes (sagittal and coronal) and a horizontal or short-axis plane (called "axial" in Seg3D). The 3 planes are orthogonal to the Cartesian axes (X, Y and $\mathrm{Z}$, respectively). It also allows one to overlay a binary segmentation mask on the image volume, and add landmarks manually using a "Brush Tool". The segmentation can be rendered and visualized in 3D. Finally, it is possible to export the segmentation mask, either as an image file, or as a . mat file that can be loaded into Matlab for off-line processing.

\section{B. Slice-by-slice manual segmentation}

We used the Seg3D Brush Tool to emulate the slice-byslice manual segmentation process from the literature. An expert scrolled through the image using the 3 main planes, and for each slice placed landmarks on the annulus. Once the expert was trained, segmentation of four valves in a single rat took for more than $2 \mathrm{~h}$, and was found to be a tedious process. An example of a slice-by-slice segmentation is shown in Fig. 1a.

Slice-by-slice segmentation was slow because the expert had to quickly scroll back and forth to use landmarks in adjoining slices as visual hints on where to place new ones. In some cases, an artifact was picked as a visual clue, and the error propagated until being noticed and corrected. The process became especially difficult in sections of the annulus that were almost parallel to one of the orthogonal planes, because the intersection of the contour with the plane turned from a well defined spot to an irregular segment. The pulmonary valve turned out to be a particularly hard challenge, as parts of its annulus contour are rather parallel to the long-axis planes. Additional image artifacts appeared due to the pulmonary artery having been nipped close to the annulus in the wet lab preparation, causing distortion to the valve.

\section{Manual segmentation with sparse set of landmarks}

The over $2 \mathrm{~h}$ spent in the slice-by-slice segmentation were shortened by instructing the expert to place only a sparse set of landmarks. The sparse set of landmarks was then sorted and interpolated with a spline curve. Lei et al. [16] proposed a similar idea for the mitral annulus, limiting the

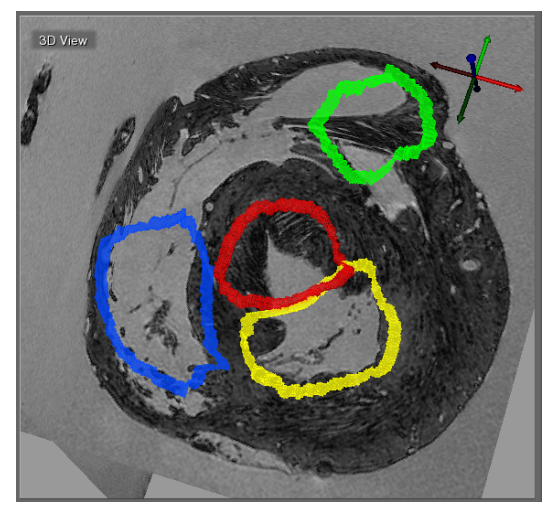

(a) Manual slice-by-slice segmentation.

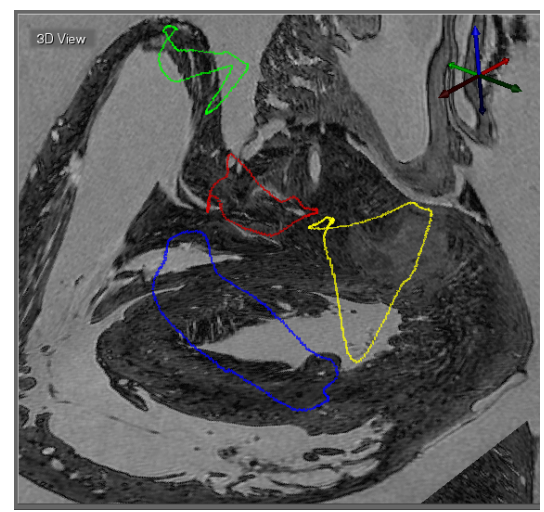

(b) Spline Tool assisted segmentation.

Fig. 1: Manual segmentation of the annulae of the four main cardiac valves: Aortic (red), mitral (yellow), pulmonary (green), tricuspid (blue). Annulae isosurfaces are displayed floating in the Seg3D 3DView window. The grayscale background corresponds to the MR image intersected by the 3 orthogonal planes.

number of two-dimensional (2D) echocardiography angular acquisitions to 10, hand-tracing only 2 annulus points in each of them. The authors proposed a method called Connected Equi-Length Curve Angle Chain (CELCAC) to cluster the landmarks into segments, and sort the segments according to their curve direction. Finally, the sorted points were interpolated with a non-uniform rational B-spline (NURBS) curve.

Our approach differs from this in some aspects. First, our experts can freely scroll the image volume and choose the slices of interest. Second, the landmarks are sorted with a simpler and fast elevation invariant azimuth sorting method described in the next section. Third, the sorted points are interpolated using a natural cubic spline with centripetal parameterisation [5]. This is the same interpolation algorithm implemented in Matlab's function cscvn ().

In preliminary tests (30 min for each whole heart), the sparse set of landmarks method was found to be error prone, as the lack of visual context from adjacent slices gave rise to segmentation landmarks being placed at spurious locations. It was also very hard for the expert to evaluate the quality of the segmented annulus by looking at the $3 \mathrm{D}$ reconstruction 
of the landmarks alone. This agrees with a recent publication in semi-automatic segmentation of the annulus of the mitral valve, where the authors noted that manual segmentations using 2D slices "only allow access to a portion of the available information at each step. This requires the user to mentally interpolate the annulus surroundings, and often results in a lack of spatial coherence" [18]. Mental interpolation is made more difficult by the fact that the annulus is not planar, as discussed in section I.

\section{Elevation invariant azimuth sorting method}

Let the 3D coordinates of the $i$-th landmark be the column vector $s_{i}=\left[\begin{array}{lll}X_{1 i}, & X_{2 i}, & X_{3 i}\end{array}\right]^{\top}$. Let the training set of $M$ landmarks be the matrix $S=\left[s_{1}, \ldots, s_{M}\right]$. The eigenvectors $V=\left[v_{1}, v_{2}, v_{3}\right]$ of the set of landmarks are computed using Principal Component Analysis (PCA); that is, as solutions to the eigenproblem $C v=\lambda v$, where $\lambda$ is an eigenvalue, and $C$ is the covariance matrix $C=$ $\frac{1}{M} \sum_{i=1}^{M}\left(s_{i}-\bar{s}\right)\left(s_{i}-\bar{s}\right)^{\top}, \bar{s}=\frac{1}{M} \sum_{i=1}^{M} s_{i}$.

The 2 eigenvectors with the largest eigenvalues, $v_{1}, v_{2}$, span the best approximation to an ideal "valve plane" (Fig. 2a). The landmark coordinates $s_{i}$ can be rotated so that the eigenvectors align with the Cartesian axes by simply doing $s_{i}^{\prime}=V^{\top} s_{i}$. This effectively turns the valve "horizontal" (Fig. 2b), and makes the method invariant to similarity transformations (scaling, rotation, translation), and thus to the valve's plane elevation. Finally, the azimuth value to the origin is computed for each rotated and centered landmark (Fig. 2c), and used to order the landmarks.

\section{E. Spline Tool for computer assisted segmentation}

In order to add real time visual feedback to the sparse set of landmarks method, we developed a new Spline Tool for the Seg3D platform, using an existing Polyline Tool as a starting point.

The Spline Tool allows an expert to add, move and delete landmarks to the image volume. The landmarks are used as control points for a 3D interpolating natural cubic spline. The spline can be visualized on the orthogonal planes in two ways: A) as a $2 \mathrm{D}$ projection on the plane; B) as the intersection with the plane. Visualization B (Fig. 3) is more useful for refining the current spline by adding new landmarks, while visualization $\mathrm{A}$ is more useful for finding spurious landmarks added by accident.

We propose the following segmentation protocol: 1) Move the planes to the approximate center of the annulus. 2) Zoom in and adjust the contrast and intensity of the image for optimal tissue visualisation. 3) Place two landmarks on the sagittal plane, and two landmarks on the coronal plane. 4) Scroll back and forth with the long-axis planes looking for locations where the current spline does not follow the annulus, and that are roughly half way between two existing landmarks. 5) Add a new landmark on the annulus to refine the spline. 6) Repeat steps 4) and 5) as necessary.

The segmentation can be be visualized in 3D (Fig. 1b) and exported as a binary mask. The spline control points can also be exported as a text file for further processing.

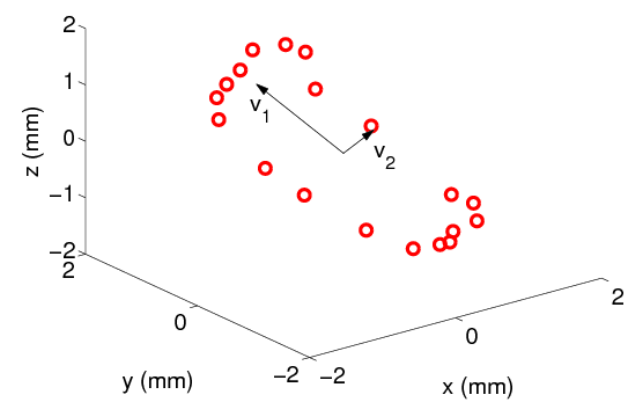

(a) Segmentation landmarks (red circles) hand traced on the annulus, and PCA eigenvectors with 2 largest eigenvalues, $v_{1}, v_{2}$.

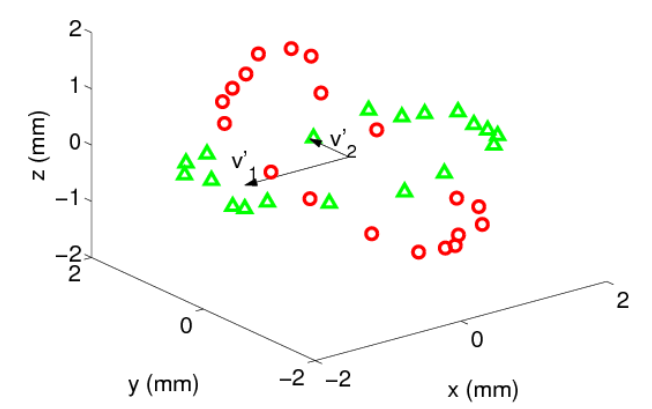

(b) Segmentation landmarks rotated (green triangles) in 3D to align eigenvectors with Euclidean axes.

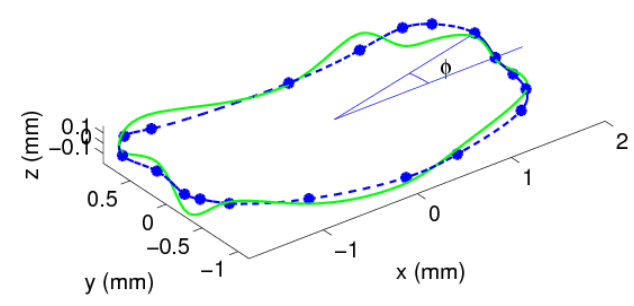

(c) Rotated and centered landmarks projected on horizontal plane (blue asterisks), azimuth angle $\phi$, interpolating 3D spline after azimuth ordering (green solid curve), spline projected on horizontal plane (dashed blue curve).

Fig. 2: Elevation invariant azimuth sorting method applied to $M=19$ landmarks on a tricuspid annulus.

\section{EXPERIMENTS}

For qualitative analysis, the annulae of the four valves from 14 rats were segmented by two experts using the new Spline Tool by the elevation invariant sorting method. The experts placed landmarks on the annulus to refine the spline until they considered they could not improve the segmentation. The experts were satisfied with the results except for two cases of the pulmonary annulus. In both cases, the elevation invariant azimuth method failed to sort the landmarks in the right order, due to the valve being warped. Segmentation times decreased from more than $2 \mathrm{~h}$ to $15 \mathrm{~min}$ per heart, except in the case of difficult pulmonary annulae, that typically required an extra $15 \mathrm{~min}$ to complete. The experts reported that segmentation had become much more agreeable compared to the slice-by-slice approach. 


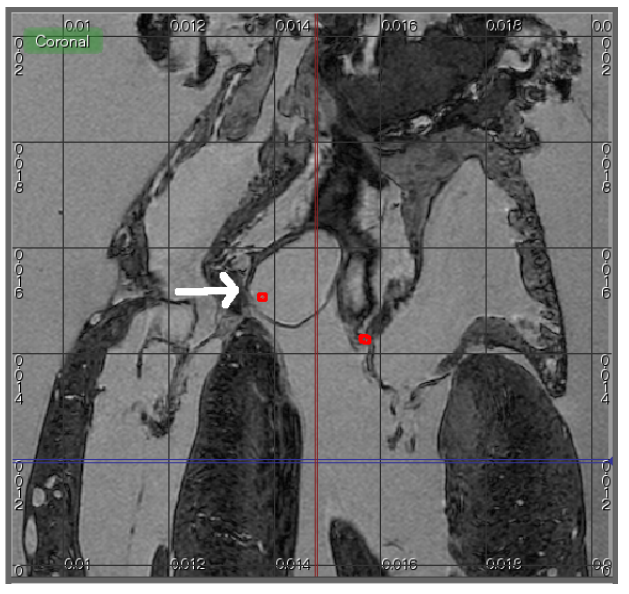

Fig. 3: Spline Tool example showing the coronal plane at the aortic annulus. Red dots provide visual feedback by showing the intersection of the current 3D spline with the plane. The red dot on the left away from the annulus indicates that the current spline needs to be refined with a new landmark. We have overlayed a white arrow to point out the position for the new landmark.

\section{CONCLUSIONS AND FUTURE WORK}

In this paper we have discussed the importance of manual annulus segmentation for the four main valves of the heart. We have reviewed two traditional manual segmentation approaches, slice-by-slice and interpolating a sparse set of landmarks with a spline curve. We have developed a new Spline Tool for the open source software platform Seg3D [19], that is fast and improves spatial coherence by providing visual feedback of the segmentation in real time. The Spline Tool was tested successfully on 14 rat hearts.

The database of manually segmented annulae will be used for shape and inter-expert variability analysis. It could also be used for validation of semi-automatic and automatic segmentation methods. In future work, we will apply the same tools and methodology to human data.

To address current issues with anatomical distortions, improvements in the wet lab protocols are necessary. The insertion of a cannula into the cut pulmonary artery, prior to fixation may prevent the pulmonary artery from collapsing.

Currently the Spline Tool is available to the wider community from a branch of the Seg3D code. We understand that it will be integrated into the main development branch soon.

\section{ACKNOWLEDGMENTS}

The authors thank the financial support of the BBSRCfunded Oxford 3D Heart Project (BB E003443). RC is a postdoctoral researcher in project preDiCT (EC FP7). TAQ is an EPSRC Postdoctoral Fellow. PK is a senior Fellow of the British Heart Foundation. VG is a Research Councils UK Fellow. This work was made possible in part by software from the NIH/NCRR Center for Integrative Biomedical
Computing, P41-RR12553-10; and by the spline computation software in the Qwt project (http://qwt.sf.net). The authors also want to thank Kristen Zygmunt, Michael Callahan, Ayla Khan and Allen from the Seg3D project for their help with the implementation of the Spline Tool.

\section{REFERENCES}

[1] J.A. Ormiston et al., "Size and motion of the mitral valve annulus in man. II. Abnormalities in mitral valve prolapse", Circulation, 65(4):713-719, 1982.

[2] C. Tei et al., "The tricuspid valve annulus: study of size and motion in normal subjects and in patients with tricuspid regurgitation", Circulation, 66:665-671, 1982.

[3] R.A. Levine et al., "The relationship of mitral annular shape to the diagnosis of mitral valve prolapse", Circulation, 75(4):756-767, 1987.

[4] R.A. Levine et al., "Three-dimensional echocardiographic reconstruction of the mitral valve, with implications for the diagnosis of mitral valve prolapse”, Circulation, 80(3):589-598, 1989.

[5] E.T.Y. Lee, "Choosing nodes in parametric curve interpolation", Computer-Aided Design, 21:363-370, 1989.

[6] K.A. Powell et al., "3-D reconstruction of mitral annulus from 2-D transesophageal echocardiographic images", in Computers in Cardiology, 1994.

[7] S. Chandra et al., "Three dimensional reconstruction (shape and motion) of tricuspid annulus in normals and in patients after tricuspid annuloplasty with a flexible ring", in Computers in Cardiology, 1996, pp. 693-696.

[8] Y. Yamaura et al., "Evaluation of the mitral annulus by extracted three-dimensional images in patients with an annuloplasty ring", The American J. of Cardiology, 82(4):534-536, 1998.

[9] R.H. Anderson, "Clinical anatomy of the aortic root", Heart, 84:670 673, 2000.

[10] S.R. Kaplan et al., "Three-dimensional echocardiographic assessment of annular shape changes in the normal and regurgitant mitral valve", American Heart J., 139(3):378-387, 2000.

[11] I. Wolf et al., "Three-dimensional annulus segmentation and hybrid visualisation in echocardiography", in Computers in Cardiology, 2001, pp. 105-108.

[12] J.E. Schneider et al., "Identification of cardiac malformations in mice lacking Ptdsr using a novel high-throughput magnetic resonance imaging technique". BMC Dev Biol, 4(1):16-27, 2004.

[13] Y. Shang et al., "Prior Based Cardiac Valve Segmentation in Echocardiographic Sequences: Geodesic Active Contour Guided by Region and Shape Prior", in Pattern Recognition and Image Analysis, 2005, pp. 447-454.

[14] R.A.B. Burton et al., "Three-dimensional models of individual cardiac histo-anatomy: tools and challenges", Ann NY Acad Sci, 1080:301319, 2006.

[15] J. Kwan et al., "3D geometry of a normal tricuspid annulus during systole: a comparison study with the mitral annulus using real-time 3D echocardiography", European J. of Echocardiography, 8(5):375383, 2007.

[16] Z. Lei et al., "Three Dimensional Reconstruction and Dynamic Analysis of Mitral Annular Based on Connected Equi-length Curve Angle Chain", in International Conference on Medical Biometrics, 4901:298-306, Springer Berlin/Heidelberg, 2007.

[17] G. Plank et al., "Generation of histo-anatomically representative models of the individual heart: tools and application", Phil. Trans. Series A, Math., phys., and eng. sciences, 367(1896):2257-2292, 2009.

[18] R.J. Schneider et al., "Mitral annulus segmentation from threedimensional ultrasound", in ISBI '09, Boston, USA, 2009, pp. 779782 .

[19] Seg3D: Volumetric Image Segmentation and Visualization. Scientific Computing and Imaging Institute (SCI), http: //www. seg3d.org. Last visited: 24 June 2010. 